\section{The Protective Effect of Exogenous Putrescine in the Response of Tea Plants (Camellia sinensis) to Salt Stress}

\author{
Fei Xiong, Jieren Liao, Yuanchun Ma, Yuhua Wang, Wanping Fang, \\ and Xujun $\mathrm{Zhu}^{1}$ \\ College of Horticulture, Nanjing Agricultural University, Nanjing 210095, \\ People's Republic of China
}

Additional index words. physiological metabolism, Camellia sinensis, salt stress, putrescine

\begin{abstract}
Camellia sinensis cultivars were treated with $5 \mathrm{~mm}$ putrescine (Put) under a range of sodium chloride $(\mathrm{NaCl})$ concentrations. Plant performance, as indicated by various indicators associated with plant growing condition such as photosynthetic parameters and polyamine (PA) contents, especially the content of Put, was improved by the treatment. The extent of both $\mathrm{Na}^{+}$accumulation and $\mathrm{K}^{+}$loss increased. The activity levels of the antioxidant enzymes related to salt stress, such as superoxide dismutase (SOD), peroxidase (POD), and catalase, were significantly altered with different salt stress levels and showed a decrease in the general trend. Besides, tea polyphenols, the tea quality indicator, increased during the salinity treatment but decreased when we applied Put. These findings suggest that treatment with Put might constitute an effective means for alleviating salinity stress-induced injury through its positive effect on photosynthetic efficiency and for controlling reactive oxygen species (ROS) scavenging ability under appropriate salt stress levels.
\end{abstract}

The salt-sensitive tea plant $C$. sinensis (L.) O. Kuntze is naturally distributed in the Southeast Asian monsoon region and is a commercially valuable plant, particularly in China, where it has been cultivated for at least 2000 years (Chen et al., 2009). In some regions of the world, salinity is the most serious threat to agriculture and the environment (Golldack et al., 2014). Salt stress can cause osmotic stress, ion poisoning, and secondary stresses (secondary stresses, such as nutritional deficiency and oxidative stress, are caused by osmotic stress and ion poisoning) (Zhu, 2001). Salt stress can also destroy the cell membrane, impair its function, and disturb cell metabolism and normal physiological functioning. High salt concentrations in the soil will result in plant nutritional deficiencies. This is because competition from salt ions reduces the absorption of nutrient elements, such as potassium $\left(\mathrm{K}^{+}\right)$, resulting in $\mathrm{K}^{+}$deficiency in the plant cells (Hasegawa et al., 2000). Furthermore, salt stress can seriously disrupt the integrity of the cell membrane structure. In addition, ion poisoning can also bring negative effect on

Received for publication 8 June 2018. Accepted for publication 12 July 2018.

This research was supported by the Fundamental Research Funds for the Central Universities (KYZ201842; KYZ201841), the earmarked fund for China Agriculture Research System (CARS-19), Jiangsu Agricultural Industry Technology System (JATS[2018]280), the National Natural Science Foundation of China (31870680, 31770733), and Jiangsu Agriculture Science and Technology Innovation Fund (CX(17)2018).

${ }^{1}$ Corresponding author. E-mail: zhuxujun@njau. edu.cn. the photosynthetic system, causing a decrease in photosynthetic efficiency, and metal ions degrade the cell performance by disturbing the ionic transport through the polymer membrane (Ehteshami et al., 2016). However, it is not known how this actually occurs in $C$. sinensis. Secondary stress causes various deleterious effects in $C$. sinensis, including yellowing and wilting of the leaves, as well as reduced leaf growth; hindering of root growth; decreases in the photosynthetic rate; and increases in energy consumption, eventually accelerating the aging of plants and even causing death (Yang et al., 2006). To deal with ionic and osmotic stresses, plants have evolved a series of strategies to minimize the toxic effects of salt stress. Oxidative stress, as one of the strategies, is invoked under salinity stress as an early stress response and is associated with the generation of ROS (Halliwell, 2006). Under normal circumstances, the stable content of ROS in plants is at a level harmless to the plant, but salt stress disrupts this stable equilibrium (Wang et al., 2004), causing peroxidation and destruction of the membrane system and metabolic functioning, leading to further damage to biological macromolecules, such as proteins and nucleic acids, ultimately resulting in cell death (Mittler, 2002). To mitigate and cope with the damages initiated by ROS, plants have evolved a complex antioxidative system that includes enzymatic antioxidants (Scandalios, 1993).

Polyamines, which mainly include spermidine (Spd), spermine (Spm), and Put, are small polycations observed in various bacteria and plants, occurring ubiquitously in plant tissues. It was recently reported that PAs function in growth and differentiation processes in plants, as well as in the plant response to any stress condition (Gill and Tuteja, 2010). The primary material of the PA biosynthetic pathway is Put (Slocum et al., 1984). Following Put biosynthesis, methionine donates ammonia propyl, resulting in the gradual formation of Spd and Spm. The PA transport system has feedback regulation mechanisms in the cells (Khan et al., 1991). Free PA levels in plant cells depend not only on their synthesis but also on their transport, degradation, and conjugation (Groppa and Benavides, 2008). Under salt stress, applying an appropriate concentration of exogenous PAs can alleviate the effect of salt to a certain extent (Groppa and Benavides, 2008). A previous study showed that exogenous Put treatment improved the $\mathrm{K}^{+} / \mathrm{Na}^{+}$ratio in broad bean under $\mathrm{NaCl}$ stress and could alleviate part of the damage resulting from the accumulation of $\mathrm{Na}^{+}$and $\mathrm{Cl}^{-}$(Zeid, 2004). PAs, under physiological conditions, exist in the form of cationic polymers. In addition, PAs exhibit features of combined with low molecular or polymer in the cell, also can be used as small-molecule osmotic solutes to directly regulate osmotic adjustment (Masson et al., 2017). Bigo et al. (2001) reported that PAs can be covalently bonded with thylakoid membrane proteins under glutamine transferase catalysis and play an important role in the process of plant adaptation to salt stress. Another study (Tang and Newton, 2005) reported that PAs, especially Put, reduce callus stress and lipid peroxidation damage in pine seedlings subjected to salt stress by improving antioxidant enzyme activity. In summary, the positive role of PAs in stress relates to their influence on membrane structure stability, ROS scavenging capacity, their reaction with large biological molecules, and their role in signal transduction. Under salt stress, PA contents and varieties in plants change rapidly, which alters related physiological and biochemical reactions in plants and adjusts their resistance to salt stress (Tonon et al., 2004). The adaptation of plants to salt stress is not only associated with the PA content but also relates to the interaction between PAs. A study has shown that the ratio of $(\mathrm{Spm}+\mathrm{Spd}) /$ Put under salt stress only increases in strongly salt-resistant tomatoes, not in saltsensitive varieties, suggesting that a high level of accumulation of Spd and Spm can alleviate salt stress damage (Santacruz et al., 1997). Salt stress makes changes in PA contents related to salt resistance closely in plants has been basically reached a consensus. But the physiological function of Put is also unclear under salt stress and requires further research. Furthermore, no studies have investigated the role of exogenous Put in C. sinensis under salt stress. In the present work, we tested the hypotheses that 1) the growth of $C$. sinensis can be protected by exogenous Put on a short-term basis, even after only a few days of treatment, and 2) the impact of exogenous Put on salt-treated $C$. sinensis depends on the concentration of salt in relation to the influence of exogenous Put on endogenous PA metabolism. 


\section{Materials and Methods}

Plant material and chemical treatments. Cutting seedlings of 2-year-old tea plants (Longjing-changye) with one bud and seven or eight leaves were grown in a chamber at the Nanjing Agricultural University (Nanjing, China). We chose plants with consistent growth as treatment materials. The plants were grown in Hoagland nutrient solution (HS) (pH 5.6), and the growth conditions were maintained at $23 \pm 2{ }^{\circ} \mathrm{C}$ temperature and $70 \% \pm 10 \%$ relative humidity. Salinity stress was imposed by adding $50 \mathrm{~mm} \mathrm{NaCl}$ and $100 \mathrm{~mm} \mathrm{NaCl}$ to the HS, whereas the control treatment comprised only the HS. To deliver Put, four foliar applications were given, Put solution was made up in water (pH 9.20). The Put concentration $(5 \mathrm{~mm})$ was determined through preliminary experiments and referred to Yiu et al. (2009). The treatment was started on the first day of salinity stress and repeated every other day. One bud and two leaves of the plants were harvested 1 and $8 \mathrm{~d}$ after treatment; some samples were frozen in liquid nitrogen and stored at $-80{ }^{\circ} \mathrm{C}$ until further use, whereas the remaining leaves were dried and crushed into tea powder. The tea powder was subjected to six distinct treatments of HS with the addition of 1) $0 \mathrm{~mm} \mathrm{NaCl} / 0 \mathrm{~mm}$ Put, 2) $0 \mathrm{~mm} \mathrm{NaCl} /$ $5 \mathrm{~mm}$ Put, 3) $50 \mathrm{~mm} \mathrm{NaCl} / 0 \mathrm{~mm}$ Put, 4) $50 \mathrm{~mm}$ $\mathrm{NaCl} / 5 \mathrm{~mm}$ Put, 5) $100 \mathrm{~mm} \mathrm{NaCl} / 0 \mathrm{~mm}$ Put, and 6) $100 \mathrm{~mm} \mathrm{NaCl} / 5 \mathrm{~mm}$ Put.

$P A$ extraction and tissue content determination. The techniques for extraction of PAs and for ultra high performance liquid chromatography (UHPLC) analysis were described by Zhu et al. (2015) with slight modifications. The modifications are as follows: n-butyl alcohol was added together with perchloric acid for better extraction rate and then centrifuged at $3600 g_{n}$ for $5 \mathrm{~min}$, and after standing for $1 \mathrm{~min}$, the upper organic phase was transferred to a new tube for determination.

Antioxidant enzyme extraction and assays. The lyophilized leaves were used for the enzyme assays. The enzymes were extracted from $0.25 \mathrm{~g}$ lyophilized leaves with the grinding medium consisting of $50 \mathrm{~mm}$ phosphate buffer ( $\mathrm{pH} 7.8$ ), $0.1 \mathrm{~mm}$ EDTA, and $1 \%$ polyvinylpyrrolidone, and the homogenate was centrifuged at $13,000 g_{n}$ for 10 min. The supernatant was collected for the measurement of SOD and POD activities. SOD activity was assayed as described by Giannopolitis and Ries (1977), that of POD by following Pundir et al. (1999), and that of Catalase (CAT) by following Aebi (1984).

Determination of ion $\left(\mathrm{Na}^{+}, \mathrm{K}^{+}\right.$, and $\left.\mathrm{Ca}^{2+}\right)$ content. $\mathrm{Na}^{+}, \mathrm{K}^{+}, \mathrm{Ca}^{2+}$ determination method of extracting reference Wada et al. (2017), slightly improved. The leaves were quickly rinsed in sterile deionized water to remove ions from the free space and gently blotted dry with a paper towel. For the ions quantification, the leaves were oven-dried at $80^{\circ} \mathrm{C}$ for $4 \mathrm{~h}$ and $0.5 \mathrm{~g}$ dry weight was digested. $\mathrm{Na}^{+}, \mathrm{K}^{+}$, and $\mathrm{Ca}^{2+}$ were resuspensed in $5 \mathrm{~mL}$ of $75 \% \mathrm{HNO}_{3}$ and the solutions were filtered. The ions were quantified by an Optima 2100DV inductively coupled plasma emission spectrometer (PerkinElmer, Waltham, MA).

Determination of the tea quality indicators. The compositions of tea polyphenols and caffeine in leaves were determined by highperformance liquid chromatography (SPD20A; SHIMADZU, Kyoto, Japan) analysis, and the techniques for extraction described by Doi et al. (1981) were followed.

Three hundred milligrams (dry weight) of tea leaves (finely powdered) was extracted with $40 \mathrm{~mL}$ of distilled water in a boiling water bath for $45 \mathrm{~min}$. After centrifugation at $10,000 g_{\mathrm{n}}$ for $10 \mathrm{~min}$, the supernatant was removed and the volume made up to $50 \mathrm{~mL}$ with distilled water. Total content of free amino acids was determined by the ninhydrin method. Ten milliliters of supernatant was obtained and mixed with $5 \mathrm{~mL}$ of $2 \%$ ninhydrin reagent and $5 \mathrm{~mL}$ of $\mathrm{pH} 8.0$ phosphate buffer. The reaction mixture was heated in a boiling water bath for $15 \mathrm{~min}$. After cooling to room temperature, $5 \mathrm{~mL}$ of distilled water was added. The mixture was allowed to stand for $10 \mathrm{~min}$. Absorbance was recorded at $570 \mathrm{~nm}$ (MetashUV-5200 ultraviolet-vis spectrophotometer; Shanghai Metash Instruments Co., Ltd., Shanghai, China). L-Glutamicacid was used to make the standard curve. Each amino acid content was determined as described by Mei et al. (2016).

Measurements of chlorophyll fluorescence parameters. Leaves were adapted in the dark for $30 \mathrm{~min}$ before measurement of the chlorophyll fluorescence transient. Each measured O-J-I-P induction curve was analyzed according to the JIP test (Strasser and Strasser, 1995). PAM-2100 portable optical instrument (Walze, Germany) was used to measure the photochemical chlorophyll fluorescence parameters: efficient photosynthesis (PIABS), photochemical efficiency (Fv/Fm), photosystem II (PSII) potential photosynthetic rate, and electron transport activity ( $\psi \mathrm{O}, \varphi \mathrm{Eo}$, and $\varphi \mathrm{Po})$. Fully expanded leaves at the third or fourth position from the seedling apex were used for these determinations.

Statistical analysis. Each value was expressed as the mean \pm SE of the three independent experiments. All data were analyzed using SPSS 20.0 (Windows). Significance was determined by Duncan's test and analysis of variance. A probability level of $5 \%(P \leq 0.05)$ was considered as statistically significant.

\section{Results}

Effects of exogenous Put on endogenous $P A$ levels under salt stress. Following exposure to salinity stress, there were no significant changes in the PA content in both salinity stress treatments compared with the control by day 1 . By day 8 , the PA content showed a contrasting trend in both salinity stress treatments (Fig. 1). We noticed that the $(\mathrm{Spd}+\mathrm{Spm}) /$ Put ratio decreased under the $50 \mathrm{~mm} \mathrm{NaCl}$ treatment and increased under the $100 \mathrm{~mm} \mathrm{NaCl}$ treatment at both days 1 and 8 .

As shown in Fig. 1, a substantial increase was observed in the Put content at the exogenous Put treatment by day 1, including the cultivar under the salinity stress. But compared with the salinity treatment without exogenous Put, endogenous Put did not show the significant change. Besides, the Put content of the cultivar with $50 \mathrm{~mm} \mathrm{NaCl}$ and $5 \mathrm{~mm}$ Put treatment at day 8 have the same level compared with that at day 1 , but with a large increase at day 8 with $100 \mathrm{~mm} \mathrm{NaCl}$ and $5 \mathrm{~mm}$ Put. The reason may be that the salt stress with $50 \mathrm{~mm} \mathrm{NaCl}$ has already been relieved by the application of Put at day 8 , so the level of Put content reaches back to normal gradually, and the CAT activity (Fig. 2) supported the point. By day 8, the content of Spd declined slightly under the treatments of $50 \mathrm{~mm} \mathrm{NaCl}, 100 \mathrm{~mm} \mathrm{NaCl}$, and exogenous Put, whereas the content of Spm increased slightly at the exogenous Put treatment by day 8 .

Effects of exogenous Put on antioxidant enzyme activity under salt stress. After $1 \mathrm{~d}$ of exposure to salinity, the SOD and CAT activity levels increased by $13.9 \%$ and $8.1 \%$, respectively, whereas the POD activity level decreased by $48.8 \%$ under $50 \mathrm{~mm} \mathrm{NaCl}$. The SOD activity level increased by $4.6 \%$, whereas the CAT and POD activity levels decreased by $37.1 \%$ and $80.5 \%$, respectively, under $100 \mathrm{~mm} \mathrm{NaCl}$. After $8 \mathrm{~d}$ of exposure to salinity, the SOD activity level increased by $7.1 \%$, whereas the CAT and POD activity levels decreased by $4.3 \%$ and $13.8 \%$, respectively, under $50 \mathrm{~mm} \mathrm{NaCl}$. The SOD activity level increased by $1.9 \%$, whereas the CAT and POD activity levels decreased by $47.1 \%$ and $80.4 \%$, respectively, under $100 \mathrm{~mm} \mathrm{NaCl}$. From day 1 to day 8 , the CAT showed a significant increase at the higher salt concentration (Fig. 2).

In the presence of Put, the level of SOD activity at salt concentrations of 0,50 , and $100 \mathrm{~mm}$ increased by $3.4 \%, 2.5 \%$, and $6.7 \%$, respectively, by day 1 in comparison with the control. But at day 8 , the SOD activity showed no significant change at $0 \mathrm{~mm}$ salt concentration with Put application, whereas it decreased by $18.2 \%$ at $50 \mathrm{~mm}$ salinity stress with Put application and increased by $2.0 \%$ at $100 \mathrm{~mm}$ salt concentration with Put. The activity level of CAT under 0,50 , and $100 \mathrm{~mm}$ decreased by $44.4 \%, 70.3 \%$, and $68.5 \%$, respectively, by day 1 compared with the control. The corresponding values by day 8 were $1.2 \%, 55.4 \%$, and $47.1 \%$. As for POD, the activity level under 0,50 , and $100 \mathrm{~mm}$ salt treatment with Put decreased by $68.3 \%$, $30.5 \%$, and $33.0 \%$, respectively, by day 1 . However, at day 8 , the POD activity level showed a different trend and increased by $44.8 \%$ and $19.0 \%$ at 50 and $100 \mathrm{~mm}$ salt treatments, respectively, with Put application. With a large concentration, it decreased by $69.0 \%$ without salinity (Fig. 2 )

Effects of exogenous Put on $\mathrm{K}^{+}, \mathrm{Na}^{+}$, and $\mathrm{Ca}^{2+}$ accumulation and the $\mathrm{K}^{+} / \mathrm{Na}^{+}$ratio and $\mathrm{Ca}^{2+} / \mathrm{Na}^{+}$ratio under salt stress. Salinity in plants results in several responses, including an increase in $\mathrm{Na}^{+}$concentration with a concomitant decrease in $\mathrm{K}^{+}$; a modification of the plant water status linked to the osmotic 


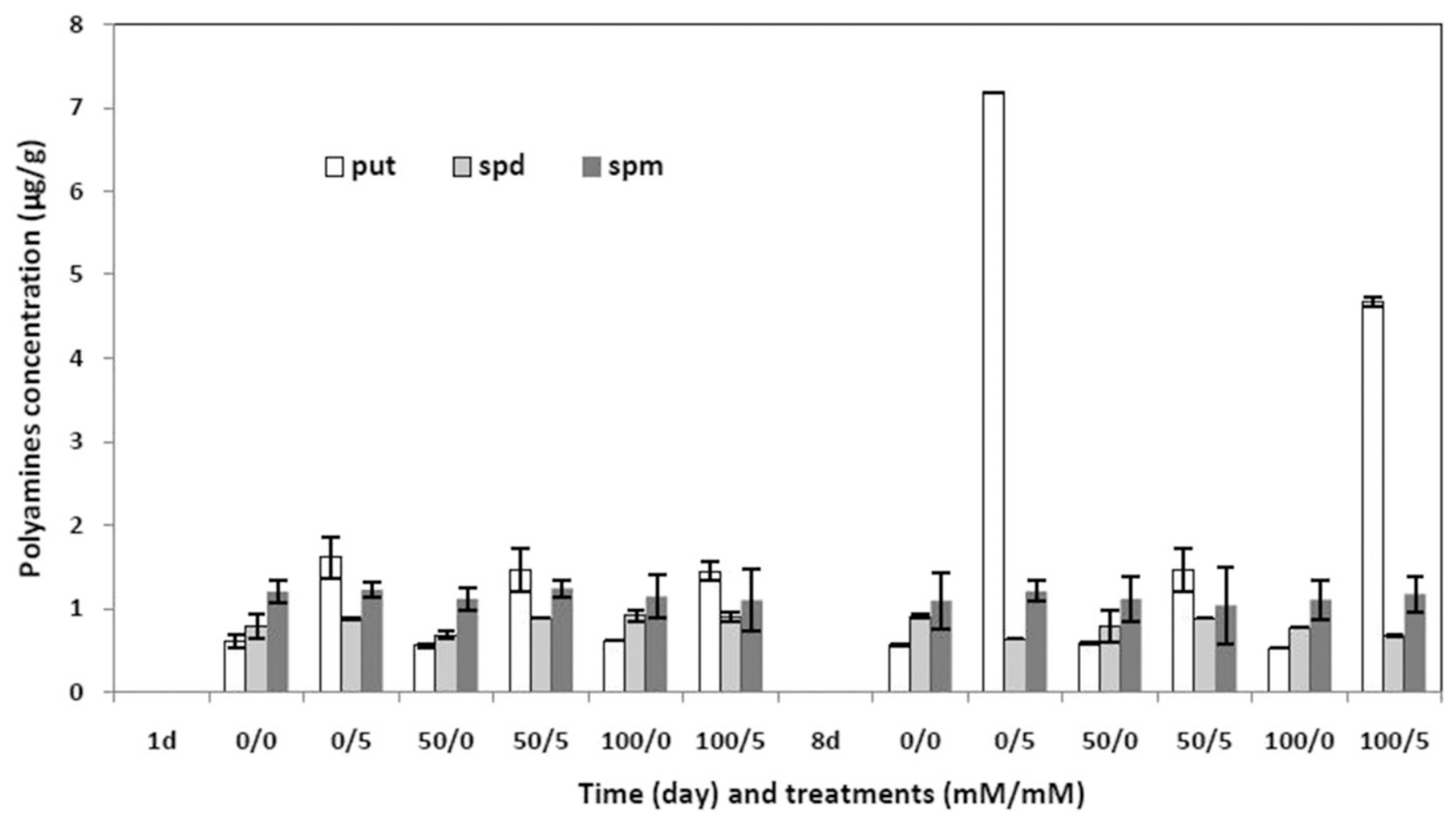

Fig. 1. The content of polyamines under exogenous putrescine (Put) application and different salinity stress $(P<0.05)$. Spd $=$ spermidine; Spm $=$ spermine.

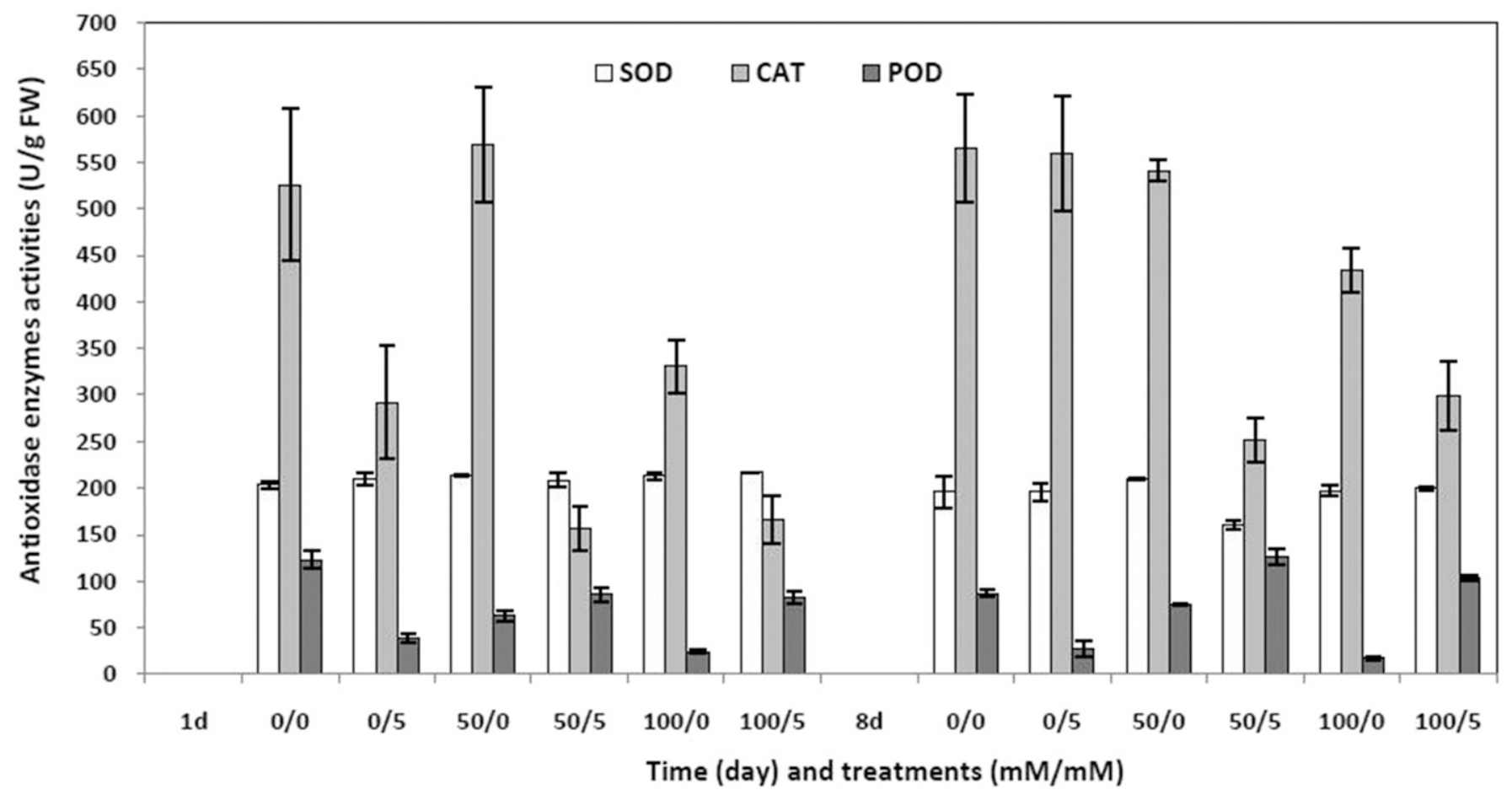

Fig. 2. Antioxidant enzyme activity under exogenous putrescine application and different salinity stress $(P<0.05)$. SOD $=$ superoxide dismutase; POD $=$ peroxidase.

component of salt stress; and secondarily induced stresses such as oxidative stress (Zhu, 2002). Calcium is important in the cell during salt stress, for example, in preserving membrane integrity, signaling in osmotic regulation, and influencing $\mathrm{K}^{+} / \mathrm{Na}^{+}$selectivity (Tozlu et al., 2000).
Compared with the control, the $\mathrm{K}^{+}$content decreased throughout the duration of the salt stress treatment. At day 1 , the $\mathrm{Na}^{+}$content declined under $50 \mathrm{~mm} \mathrm{NaCl}$ stress and increased under $100 \mathrm{~mm} \mathrm{NaCl}$ stress. However, the opposite pattern was observed by day 8 . The $\mathrm{Ca}^{2+}$ content increased under $50 \mathrm{~mm}$
$\mathrm{NaCl}$ stress but decreased under $100 \mathrm{~mm}$ $\mathrm{NaCl}$ stress. The $\mathrm{K}^{+}$content increased at higher $\mathrm{NaCl}$ concentrations but dropped at lower $\mathrm{NaCl}$ concentrations. The $\mathrm{Na}^{+}$content increased in the absence of salt treatment and increased significantly under salt treatment. The $\mathrm{Ca}^{2+}$ content dropped regardless of 
whether the plants were treated with salt or not. Compared with the control, the $\mathrm{K}^{+} / \mathrm{Na}^{+}$ ratio declined under salt stress, and the $\mathrm{Ca}^{2+} / \mathrm{Na}^{+}$ ratio dropped sharply under higher salt stress treatments (Fig. 3).

In the presence of Put, by day 1 , the $\mathrm{K}^{+}$ content in the 0,50 , and $100 \mathrm{~mm}$ salt treatments had decreased by $16.9 \%$, increased by $2.1 \%$, and decreased by $1.3 \%$, respectively. By day 8 , these values were decreased by $15.7 \%$ and $5.8 \%$, and increased by $3.2 \%$, respectively. The $\mathrm{Na}^{+}$content in the 0,50 , and $100 \mathrm{~mm}$ treatments decreased by $10.1 \%$ and increased by $58.3 \%$ and $88.2 \%$, respectively, by day 1 . It increased by $355.0 \%, 24.2 \%$, and $29.3 \%$, respectively, by day 8 . The $\mathrm{Ca}^{2+}$ content in the 0,50 , and $100 \mathrm{~mm}$ treatments decreased by $4.6 \%, 3.8 \%$, and increased by $5.7 \%$, respectively, by day 1. It increased by $4.6 \%$, decreased by $5.4 \%$, and increased by $2.2 \%$, respectively, at day 8 . From day 1 to day 8 , in comparison with the control, the $\mathrm{K}^{+}$content increased by $6.1 \%$ and decreased by $5.9 \%$ and $-2.1 \%$, respectively, at 0,50 , and $100 \mathrm{~mm}$ salt. The corresponding values for $\mathrm{Na}^{+}$content were increased by $366.9 \%, 818.6 \%$, and $855.7 \%$, and the corresponding values for $\mathrm{Ca}^{2+}$ were increased by $5.7 \%$ and decreased by $6.2 \%$ and $6.2 \%$. In the presence of Put, the $\mathrm{K}^{+} / \mathrm{Na}^{+}$ratio and $\mathrm{Ca}^{2+} / \mathrm{Na}^{+}$ ratio declined obviously under salt stress in comparison with the control (Fig. 3).

Effects of exogenous Put on tea quality indicators under salt stress. After $1 \mathrm{~d}$ of salt treatment, the free amino acid and caffeine contents decreased slightly, whereas the tea polyphenol contents evidently increased in comparison with the control. By day 8 , the caffeine and tea polyphenols had risen slightly, whereas the free amino acid content decreased at a lower salt concentration and increased at a higher salt concentration. From day 1 to day 8 , the free amino acids increased by $4.4 \%, 0 \%$, and $21.0 \%$ under 0,50 , and $100 \mathrm{~mm}$ salt treatments, respectively. The corresponding caffeine content decreased by $15.2 \%, 0 \%$, and $4.4 \%$, whereas the corresponding polyphenol content decreased by $42.3 \%$, increased by $2.9 \%$, and decreased by $0.4 \%$ (Fig. 4).

With the addition of Put, the content of free amino acids and caffeine rose gently under salt treatment, and the tea polyphenol content had dropped clearly by day 1 . By day 8 , the free amino acid content had increased with salt treatment, whereas the caffeine content had decreased. Tea polyphenols decreased at a lower salt treatment concentration but increased at a higher salt treatment concentration. From day 1 to day 8, compared with the control, the free amino acids increased by $16.7 \%, 12.3 \%$, and $0 \%$ at salt treatments of 0,50 , and $100 \mathrm{~mm}$, respectively. The caffeine content increased by $9.7 \%$ and decreased by $0.43 \%$ and $9.8 \%$ correspondingly, whereas the polyphenols decreased by $20.7 \%$ and $0 \%$, and increased by $21.1 \%$ (Fig. 4).

Impact of salt stress on photosynthetic parameters and the protection of exogenous Put under salt stress. As shown in Table 1, from day 1 to day 8 , the stress treatments reduced $\mathrm{Fv} / \mathrm{Fm}, \mathrm{Fv} / \mathrm{Fo}, \psi \circ, \varphi \mathrm{E} o, \varphi \mathrm{Po}$, and PIABS. The six parameters were reduced to $1.3 \%, 7.7 \%, 0 \%, 2.5 \%, 1.25 \%$, and $9.6 \%$, respectively, of the values achieved by plants grown under nonstressed conditions in the $50 \mathrm{~mm} \mathrm{NaCl}$ treatment, and by $12.5 \%, 39.0 \%$, $10.2 \%, 22.5 \%, 12.5 \%$, and $67.0 \%$ in the $100 \mathrm{~mm} \mathrm{NaCl}$ treatment.

The provision of $5.0 \mathrm{~mm}$ Put improved $\mathrm{Fv} / \mathrm{Fm}$, Fv/Fo, $\psi \circ, \varphi E o, \varphi$ Po, and PIABS under salt stress. For $\mathrm{Fv} / \mathrm{Fm}, \mathrm{Fv} / \mathrm{Fo}, \psi \mathrm{o}, \varphi \mathrm{Eo}, \varphi \mathrm{Po}$, and PIABS, the fold increases over the salinity treatment were, respectively, $1.03,1.09,1.14$, $1.15,1.03$, and $1.58(0 \mathrm{~mm}$ salt $) ; 1.01,1.08$, $1.10,1.13,1.01$, and $1.46(50 \mathrm{~mm} \mathrm{NaCl})$; and $1.11,1.38,1.11,1.23,1.11$, and $1.14(100 \mathrm{~mm}$ $\mathrm{NaCl})$ (Table 1).

\section{Discussion}

The effect of salinity stress on endogenous $P A$ levels. In general, plant species and cultivars with high stress tolerance have a significant capacity to maintain higher PA contents in response to environmental stress (Bouchereau et al., 1999). During salt stress, PA concentrations have been reported to both increase (Katiyar and Dubey, 1990) and decrease (Mei et al., 2016), with the ratio of $(\mathrm{Spd}+\mathrm{Spm}) /$ Put increasing with increased salinity to protect the plants under salt stress (Fariduddin et al., 2013). A previous report indicated that salt stress could enhance PA degradation (Kongkiattikajorn, 2009). In this study, the $(\mathrm{Spd}+\mathrm{Spm}) / \mathrm{Put}$ ratio decreased under the $50 \mathrm{~mm} \mathrm{NaCl}$ treatment and increased under the $100 \mathrm{~mm} \mathrm{NaCl}$ treatment over the duration of the experiment, which indicated that the tea plants of the cultivar Longjing-changye tolerate low salt stress levels. A study of the germination process in a range of species indicated that the PA composition and content were altered following salinity stress, with Put decreasing and Spd, Spm, or both increasing in most cases, which is consistent with the proposed protective function of higher PAs (Zapata et al., 2004). However, endogenous PA concentrations and contents differ in different plants (Ikbal et al., 2014). Campestre et al. (2011) showed that salinity stress reduces the endogenous Put and Spd content of soybean hypocotyls, whereas at the same time promotes that of Spm. In this study, the Put

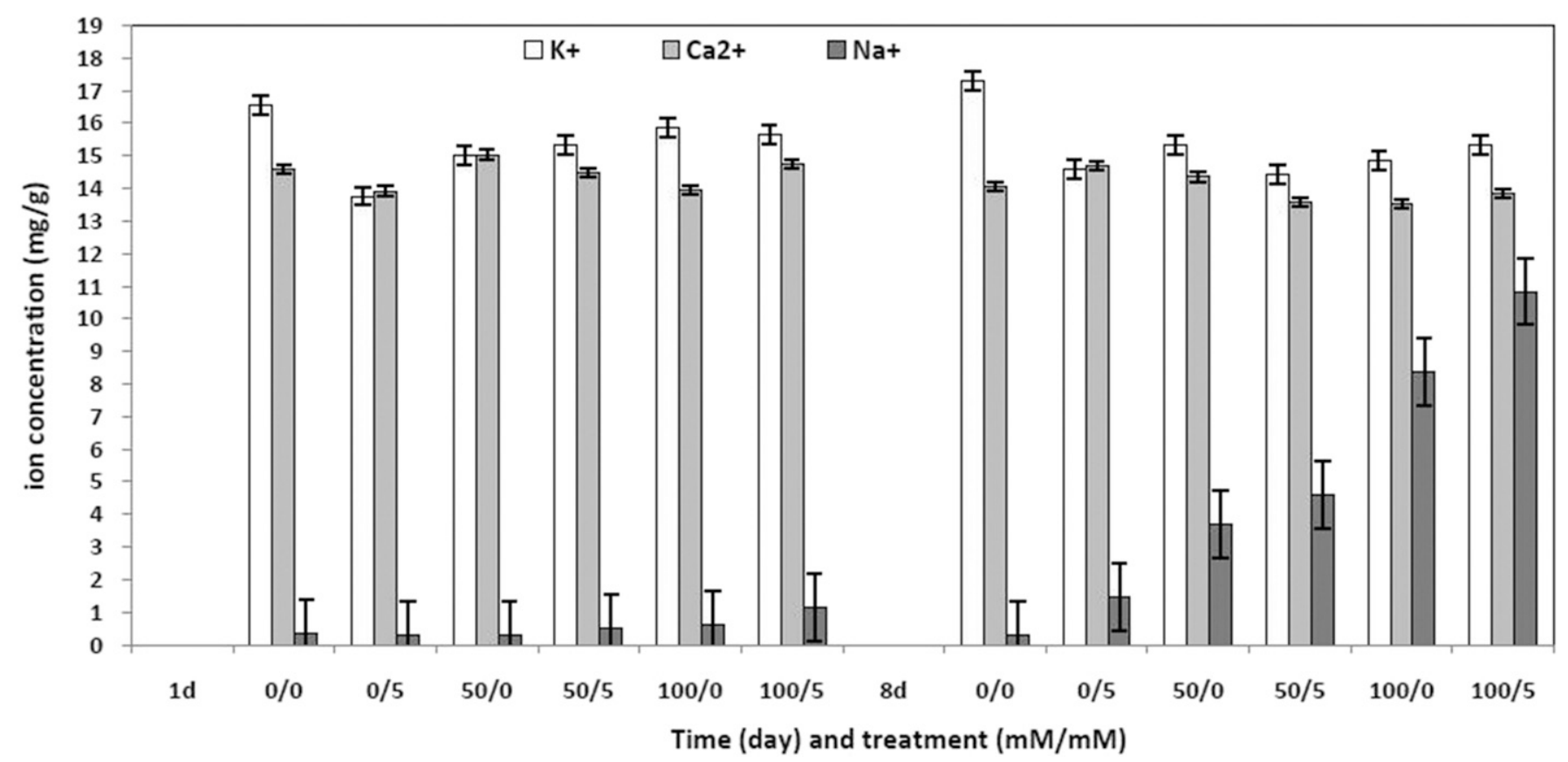

Fig. 3. $\mathrm{K}^{+}, \mathrm{Na}^{+}$, and $\mathrm{Ca}^{2+}$ accumulation under putrescine application and different salinity stress $(P<0.05)$. 


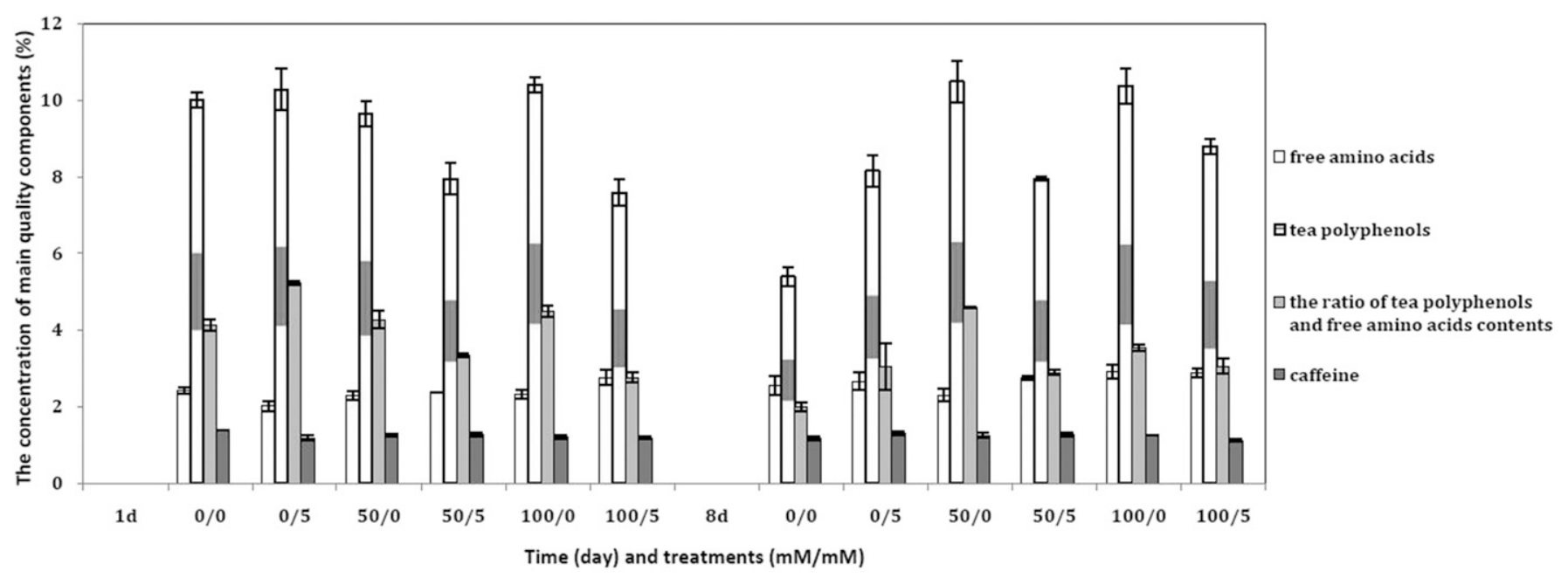

Fig. 4. The content of free amino acids, tea polyphenols, and caffeine under putrescine application and different salinity stress $(P<0.05)$.

Table 1. The chlorophyll fluorescence parameters under different treatments $(P<0.05)$.

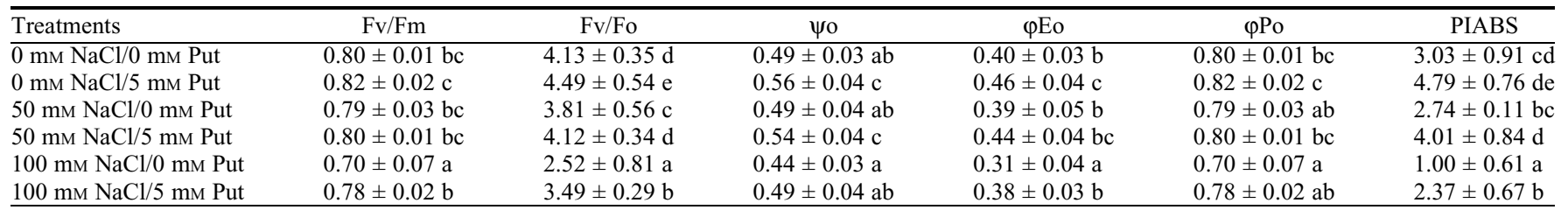

Different letters represent significant difference among six treatments.

Put $=$ putrescine.

content increased obviously under salt stress, Spd content declined after $1 \mathrm{~d}$ with $100 \mathrm{~mm}$ $\mathrm{NaCl}$ treatment, and Spm content decreased after $8 \mathrm{~d}$ of salt treatment. It is inferred that the absorption and transport mechanisms of PAs in $C$. sinensis under salt stress are very complex and are probably related to the salt level and the treatment duration.

The level of free PAs is dependent on their synthesis and on their degradation and conjugation. Soluble conjugated PAs could serve as a valuable source of free PAs that protect against stress by stabilizing protein structures and preventing protein degradation through conjugation to other proteins (Roussos and Pontikis, 2007). The accumulation of Put under salt stress may be a stress response rather than a protective mechanism (Parvin et al., 2014). Spd acts to maintain high Spm levels to improve the salt tolerance of plants (Mansour and Almutawa, 1999; Peremarti et al., 2009). Therefore, in this study, the role of the form and level of the PAs in the protective mechanism of PAs on $C$. sinensis under salt stress requires further research.

PAs in general and Put specifically are considered as an antistress agent for saltstressed plants. Salt resistance is associated with an ability to increase Put synthesis to maintain a high proportion of conjugated PAs within stressed tissues (Quinet et al., 2010). In our study, the salinity-induced changes in electrolyte leakage and photosynthesis confirmed that $C$. sinensis was compromised by salt stress. The salinity-induced generation of ROS, such as singlet oxygen, leads to increased $\mathrm{H}_{2} \mathrm{O}_{2}$ and thus lipid peroxidation, which causes membrane damage and protein and electrolyte leakage (Mudgal et al., 2010). In this study, the activity of POD and CAT decreased by day 1 and declined under the lower salt stress treatment by day 8 . This indicated that salt stress possibly influences the antioxidant system of $C$. sinensis in a short time. Key components, such as catechins, caffeine, and theanine, may be the first components used to set a discrimination standard (Obuchowicz et al., 2011). However, the quality of the tea is unclear based on the changes in the tea quality indicators.

The protective effect of the exogenous application of Put. Soil salinity is a major environmental constraint that threatens agricultural productivity. Salt stress causes ion-specific stresses resulting from changes in the $\mathrm{K}^{+} / \mathrm{Na}^{+}$ratio due to $\mathrm{Na}^{+}$uptake and $\mathrm{K}^{+}$ loss (Yamaguchi and Blumwald, 2005). However, there is a study (Ndayiragije and Lutts, 2006) which demonstrated that although Put is efficiently absorbed and translocated to the shoots and has a positive impact on monovalent cation discrimination, increased Put did not help the plant to overcome the deleterious effect of salt stress and even reinforced the negative impact of $\mathrm{NaCl}$ in terms of both shoot and root growth. In our study, it was clear that the Put treatment failed in enhancing the $\mathrm{K}^{+} / \mathrm{Na}^{+}$ratio under salt stress and even reinforced the negative impact of $\mathrm{NaCl}$ in the leaves. Indeed, Longjing-changye $C$. sinensis induced a strong accumulation of Put in the leaves and had a similar impact on $\mathrm{Na}^{+}$nutrition with exogenous Put treatment. $\mathrm{Ca}^{2+}$ content responds to salt stress and is associated with the membrane ion transfer balance (Zhang et al., 2004). Dasgan et al. (2002) found that the $\mathrm{Ca}^{2+} / \mathrm{Na}^{+}$ratio was closely related to tomato resistance to salt, even more so than the $\mathrm{K}^{+} / \mathrm{Na}^{+}$ratio. Moreover, the $\mathrm{Ca}^{2+} / \mathrm{Na}^{+}$ ratio declined in Put-treated plants under salt stress in comparison with the control. We estimated that, in the presence of Put, such a protective effect was due to the endogenous accumulation of Put and not to the competition between cations at the site of absorption.

$\mathrm{NaCl}$ inhibits seedling growth by altering the activity of antioxidant enzymes and the level of free radicals in the leaves. Put might be activating antioxidant enzymes and elevating antioxidants by controlling free radical generation, thereby preventing membrane peroxidation and the denaturation of biomolecules, ultimately improving seedling growth under salinity (Verma and Mishra, 2005). In this study, although SOD activity decreased when Put was added to the plants under salt stress, it tended to increase as the salt stress level increased. The role of Put under salinity might be to protect the superoxide radicals. $\mathrm{H}_{2} \mathrm{O}_{2}$ might be detoxified by the wellorganized functioning of the CAT and POD enzyme systems. In this study, compared with the control, CAT activity increased clearly by day 1 , and POD activity increased obviously by day 8 . Put can restrain the production of hydroxyl radicals according to the previous report (Chen and Zhang, 2000), and this could be the reason why the activity of CAT and POD decreased.

Chlorophyll fluorescence can be used as a powerful and reliable method to assess changes in the function of PSII and to examine the primary photosynthetic processes under 
environmental stress conditions (Hajiboland et al., 2010). In our results, Fv/Fm, Fv/Fo, $\psi$ o, $\varphi \mathrm{Eo}, \varphi \mathrm{Po}$, and PIABS all decreased. These results are similar to those reported by Naumann et al. (2007). Exogenous application of Put was used to compensate for salt stress, so as to confer some degree of tolerance to the photosynthetic apparatus against enhanced $\mathrm{NaCl}$ salinity and to permit cell growth (Demetriou et al., 2007). In our study, exogenous Put improved photosynthetic capacity and increased all of $\mathrm{Fv} / \mathrm{Fm}, \mathrm{Fv} / \mathrm{Fo}, \psi o, \varphi \mathrm{E} o, \varphi \mathrm{Po}$, and PIABS compared with the treatments that received $\mathrm{NaCl}$ alone. In this study, the changes in the three chemical substances, including Put, Spm, and Spd, showed no correlation when the plants under different salt stress levels were sprayed with Put. This result indicated that the relation between salt stress and the exogenous Put application treatment is unclear.

When $C$. sinensis was subjected to salt stress, the $\mathrm{Na}^{+}$content increased, resulting in ionic toxicity, whereas the $\mathrm{K}^{+}$content dropped, leading to a loss of nutrient elements. The $\mathrm{K}^{+} / \mathrm{Na}^{+}$ratio declined under salt stress, and the $\mathrm{Ca}^{2+} / \mathrm{Na}^{+}$ratio dropped sharply under the higher salt stress treatment, which damaged cell membrane integrity and stability. The increasing POD and CAT activity and the change in SOD activity were related to salt concentration, and the endogenous PA content increased to alleviate salt injury. Moreover, the impact of exogenous Put on salt-treated $C$. sinensis was cultivar dependent in terms of the influence of exogenous Put on endogenous PA metabolism. Our results illustrated that when $C$. sinensis is subjected to salt stress and exogenous Put, the $\mathrm{K}^{+} / \mathrm{Na}^{+}$ratio, $\mathrm{Ca}^{2+} / \mathrm{Na}^{+}$ratio, $\mathrm{POD}$ activity, and the endogenous PA content increased, whereas the SOD and CAT activity varied depending on the salt concentration, which relieved the secondary stress damage caused by the salt. The quality of $C$. sinensis plants under salt stress with and without exogenous Put requires further assessment.

The improvement of the $(\mathrm{Spd}+\mathrm{Spm}) / \mathrm{Put}$ ratio reflects the protective role of exogenous Spd (Fariduddin et al., 2013). Yet, the reduction of the $(\mathrm{Spd}+\mathrm{Spm}) /$ Put ratio did not reflect the protective role of exogenous Put. Although exogenous Put worsens ion poisoning, Put application alleviated salinityinduced photosynthetic inhibition, together with an increase in PA content and antioxidant enzyme activity in the leaves of $C$. sinensis. These effects varied in relation to the salt stress level and treatment duration.

\section{Literature Cited}

Aebi, H. 1984. Catalase in vitro. Methods Enzymol. 105:121-126.

Bigo, S., G. Charlet, O.B. Pardo, and J. Renaudier. 2001. Acclimation of chloroplast transglutaminase to high $\mathrm{NaCl}$ concentration in a polyaminedeficient variant strain of Dunaliella salina and in its wild type. J. Plant Physiol. 158:185-197.

Bouchereau, A., A. Aziz, F. Larher, and J. Martintanguy. 1999. Polyamines and environmental challenges: Recent development. Plant Sci. 140:103-125.
Campestre, M.P., C.D. Bordenave, A.C. Origone, A.B. Menéndez, O.A. Ruiz, A.A. Rodríguez, and S.J. Maiale. 2011. Polyamine catabolism is involved in response to salt stress in soybean hypocotyls. J. Plant Physiol. 168:1234-1240.

Chen, K. and C. Zhang. 2000. Polyamine contents in the spring wheat leaves and their relations to drought-resistance. Acta Phytophysiol. Sin. 26:381-386.

Chen, Y., M. Yu, J. Xu, X. Chen, and J. Shi. 2009 Differentiation of eight tea (Camellia sinensis) cultivars in China by elemental fingerprint of their leaves. J. Sci. Food Agr. 89:2350-2355.

Dasgan, H.Y., H. Aktas, K. Abak, and I. Cakmak. 2002. Determination of screening techniques to salinity tolerance in tomatoes and investigation of genotype responses. Plant Sci. 163:695-703.

Demetriou, G., C. Neonaki, E. Navakoudis, and K. Kotzabasis. 2007. Salt stress impact on the molecular structure and function of the photosynthetic apparatus-the protective role of polyamines. Biochim. Biophys. Acta 1767:272-280

Doi, E., D. Shibata, and T. Matoba. 1981. Modified colorimetric ninhydrin methods for peptidase assay. Anal. Biochem. 118:173-184.

Ehteshami, S.M.M., A. Taheri, and S.H. Chan 2016. A review on ions induced contamination of polymer electrolyte membrane fuel cells, poisoning mechanisms and mitigation approaches. J. Ind. Eng. Chem. 34:1-8.

Fariduddin, Q., P. Varshney, M. Yusuf, and A. Ahmad. 2013. Polyamines: Potent modulators of plant responses to stress. J. Plant Interact. 8:1-16.

Giannopolitis, C.N. and S.K. Ries. 1977. Superoxide dismutases: I. Occurrence in higher plants. Plant Physiol. 59:309-314.

Gill, S.S. and N. Tuteja. 2010. Polyamines and abiotic stress tolerance in plants. Plant Signal. Behav. 5:26-33.

Golldack, D., C. Li, H. Mohan, and N. Probst. 2014. Tolerance to drought and salt stress in plants: Unraveling the signaling networks. Front. Plant Sci. 5:151.

Groppa, M.D. and M.P. Benavides. 2008. Polyamines and abiotic stress: Recent advances. Amino Acids 34:35-45.

Hajiboland, R., N. Aliasgharzadeh, S.F. Laiegh, and C. Poschenrieder. 2010. Colonization with arbuscular mycorrhizal fungi improves salinity tolerance of tomato (Solanum lycopersicum L.) plants. Plant Soil 331:313-327.

Halliwell, B. 2006. Reactive species and antioxidants. Redox biology is a fundamental theme of aerobic life. Plant Physiol. 141:312-322.

Hasegawa, P.M., R.A. Bressan, J. Zhu, and H.J. Bohnert. 2000. Plant cellular and molecular responses to high salinity. Annu. Rev. Plant Biol. 51:463-499.

Ikbal, F.E., J.A. Hernandez, G. Barbaespin, T. Koussa, A. Aziz, M. Faize, and P. Diazvivancos. 2014. Enhanced salt-induced antioxidative responses involve a contribution of polyamine biosynthesis in grapevine plants. J. Plant Physiol. 171:779-788.

Katiyar, S. and R.S. Dubey. 1990. Changes in polyamine titer in rice seedlings following $\mathrm{NaCl}$ salinity stress. J. Agron. Crop Sci. 165:19-27.

Khan, N.A., V. Quemener, and J.P. Moulinoux. 1991. Polyamine membrane transport regulation. Cell Biol. Intl. Rpt. 15:9-24.

Kongkiattikajorn, J. 2009. Effect of salinity stress on degradation of polyamines and amine oxidase activity in maize seedlings. Kasetsart J. Natl. Sci. 43:99-117.

Mansour, M.M.F. and M.M. Almutawa. 1999. Stabilization of plasma membrane by polyamines against salt stress. Cytobios 1999:7-17.
Masson, P.H., T. Takahashi, and R. Angelini. 2017. Editorial: Molecular mechanisms underlying polyamine functions in plants. Front. Plant Sci. 8:14

Mei, X., Y. Chen, L. Zhang, X. Fu, Q. Wei, D Grierson, Y. Zhou, Y. Huang, F. Dong, and Z. Yang. 2016. Dual mechanisms regulating glutamate decarboxylases and accumulation of gamma-aminobutyric acid in tea (Camellia sinensis) leaves exposed to multiple stresses. Sci. Rpt. 6:23685

Mittler, R. 2002. Oxidative stress, antioxidants and stress tolerance. Trends Plant Sci. 7:405-410.

Mudgal, V., N. Madaan, and A. Mudgal. 2010. Biochemical mechanisms of salt tolerance in plants: A review. Intl. J. Bot. 6:136-143.

Naumann, J.C., D.R. Young, and J.E. Anderson. 2007. Linking leaf chlorophyll fluorescence properties to physiological responses for detection of salt and drought stress in coastal plant species. Physiol. Plant. 131:422-433.

Ndayiragije, A. and S. Lutts. 2006. Do exogenous polyamines have an impact on the response of a salt-sensitive rice cultivar to $\mathrm{NaCl}$ ? J. Plant Physiol. 163:506-516.

Obuchowicz, J., U.H. Engelhardt, and K. Donnelly. 2011. Flavanol database for green and black teas utilising ISO 14502-1 and ISO 14502-2 as analytical tools. J. Food Compos. Anal. 24:411-417

Parvin, S., O.R. Lee, G. Sathiyaraj, A. Khorolragchaa, Y. Kim, and D. Yang. 2014. Spermidine alleviates the growth of saline-stressed ginseng seedlings through antioxidative defense system. Gene 537:70-78

Peremarti, A., L. Bassie, P. Christou, and T. Capell. 2009. Spermine facilitates recovery from drought but does not confer drought tolerance in transgenic rice plants expressing Datura stramonium S-adenosylmethionine decarboxylase. Plant Mol. Biol. 70:253-264.

Pundir, C.S., V. Malik, A.K. Bhargava, M. Thakur, V. Kalia, S. Singh, and N.K. Kuchhal. 1999 Studies on horseradish peroxidase immobilized onto arylamine and alkyl amine glass. J. Plant Biochem. Biotechnol. 8:123-126.

Quinet, M., A. Ndayiragije, I.S. Lefevre, B Lambillotte, C.C. Dupontgillain, and S. Lutts. 2010. Putrescine differently influences the effect of salt stress on polyamine metabolism and ethylene synthesis in rice cultivars differing in salt resistance. J. Expt. Bot. 61:27192733

Roussos, P.A. and C.A. Pontikis. 2007. Changes of free, soluble conjugated and bound polyamine titers of jojoba explants under sodium chloride salinity in vitro. J. Plant Physiol. 164:895-903.

Santacruz, A., M. Acosta, F. Perezalfocea, and M.C. Bolarin. 1997. Changes in free polyamine levels induced by salt stress in leaves of cultivated and wild tomato species. Physiol. Plant. 101:341-346.

Scandalios, J.G. 1993. Oxygen stress and superoxide dismutases. Plant Physiol. 101:7-12.

Slocum, R.D., R. Kaursawhney, and A.W. Galston. 1984. The physiology and biochemistry of polyamines in plants. Arch. Biochem. Biophys. 235:283-303.

Strasser, B.J. and B.J. Strasser. 1995. Measuring fast fluorescence transients to address environmental questions: The JIP-Test, p. 977-980. In: P. Mathis (ed.). Photosynthesis: From light to biosphere. Kluwer Academic Publisher, Dordrecht.

Tang, W. and R.J. Newton. 2005. Polyamines reduce salt-induced oxidative damage by increasing the activities of antioxidant enzymes and decreasing lipid peroxidation in Virginia pine. Plant Growth Regulat. 46:31-43. 
Tonon, G., C. Kevers, O. Faivre-Rampant, M. Grazianil, and T. Gaspar. 2004. Effect of $\mathrm{NaCl}$ and mannitol iso-osmotic stresses on proline and free polyamine levels in embryogenic Fraxinus angustifolia callus. J. Plant Physiol. 161:701-708.

Tozlu, I., G.A. Moore, and C.L. Guy. 2000. Effects of increasing $\mathrm{NaCl}$ concentration on stem elongation, dry mass production, and macroand micro-nutrient accumulation in Poncirus trifoliata. Austral. J. Plant Physiol. 27:35-42.

Verma, S. and S.N. Mishra. 2005. Putrescine alleviation of growth in salt stressed Brassica juncea by inducing antioxidative defense system. J. Plant Physiol. 162:669-677.

Wada, A., N. Nonose, M. Ohata, and T. Miura. 2017. Determination of ultra-trace metal impurities in high-purity cadmium using inductively coupled plasma mass spectrometry after matrix separation with anion exchange resin. Anal. Sci. 33:357.
Wang, B., U. Lüttge, and R. Ratajczak. 2004 Specific regulation of SOD isoforms by $\mathrm{NaCl}$ and osmotic stress in leaves of C3 halophyte. J. Plant Physiol. 161:285-293.

Yamaguchi, T. and E. Blumwald. 2005. Developing salt-tolerant crop plants: Challenges and opportunities. Trends Plant Sci. 10:615-620.

Yang, Y., D.A. Jiang, H.X. Xu, C.Q. Yan, and S.R Hao. 2006. Cyclic electron flow around photosystem 1 is required for adaptation to salt stress in wild soybean species Glycine cyrtoloba ACC547. Biol. Plant. 50:586-590.

Yiu, J.C., L.D. Juang, Y.T. Fang, C.W. Liu, and S.J. Wu. 2009. Exogenous putrescine reduces flooding-induced oxidative damage by increasing the antioxidant properties of Welsh onion. Scientia Hort. 120:306-314.

Zapata, P.J., M. Serrano, M.T. Pretel, A. Amoros, and M.A. Botella. 2004. Polyamines and ethylene changes during germination of different plant species under salinity. Plant Sci. 167:781788.

Zeid, I.M. 2004. Response of bean (Phaseolus vulgaris) to exogenous putrescine treatment under salinity stress. Pak. J. Biol. Sci. 7:219-225.

Zhang, J.Z., R.A. Creelman, and J. Zhu. 2004 From laboratory to field. Using information from Arabidopsis to engineer salt, cold, and drought tolerance in crops. Plant Physiol. 135:615-621.

Zhu, J. 2001. Plant salt tolerance. Trends Plant Sci. 6:66-71.

Zhu, J. 2002. Salt and drought stress signal transduction in plants. Annu. Rev. Plant Biol. 53:247-273.

Zhu, X., Q. Li, J. Hu, M. Wang, and X. Li. 2015. Molecular cloning and characterization of spermine synthesis gene associated with cold tolerance in tea plant (Camellia sinensis). Appl. Biochem. Biotechnol. 177:1055-1068. 